\title{
Crowdsourcing Personalized Weight Loss Diets
}

\author{
Simo Hosio', Niels van Berkel2, Jonas Oppenlaender1, Jorge Goncalves² \\ ${ }^{1}$ University of Oulu, Finland \\ ${ }^{2}$ The University of Melbourne, Australia
}

\begin{abstract}
Diet is a key aspect in losing weight. Yet choosing a suitable diet among the practically unlimited options out there remains a daunting task. To this end, we contribute The Diet Explorer: a lightweight system usable with an intuitive graphical user interface, that relies on aggregated human insights for assessing and recommending suitable weight loss diets. We compared the system's performance against the de-facto online search engine, Google, in discovering personalised diets. Our results suggest that the system, bootstrapped using a public crowdsourcing platform, provides results comparable to those of Google in terms of overall satisfaction, relevance, and trustworthiness. However, The Diet Explorer was perceived as significantly faster than Google for discovering diets. Finally, this paper contributes an open-source version of the system, re-implemented as a WordPress plugin for the scientific community to use and modify for their own purposes.
\end{abstract}

Keywords: Crowdsourcing, Health-Information, Personalised Recommendations, Systems Evaluation

\section{INTRODUCTION}

Obesity is a growing health problem around the world and is described as a global epidemic by the World Health Organization (WHO). Not only is being overweight a psychologically sensitive issue, but it has been shown to negatively affect health in a plethora of ways, such as accelerating ageing or increasing the risk of diabetes and heart conditions. Examining the issue strictly through the academic lens, two aspects matter in losing weight: diet and exercise. However, previous work has shown that the former is substantially more important [1]. Yet there is no consensus even among scientists on what is the optimal diet for losing weight (think of, for example, the constant "battle" between low-carb and lowfat diets). As a result, one identified problem in the dieting space is indeed information overload [2]. There are simply too many diets to choose from.

In this paper, we present The Diet Explorer (TDE): a crowd-powered tool that exploits crowdsourcing and wisdom of the crowd [3] in first assessing and then recommending personalised weight loss diets. Using paid labour from Prolific Academic (https://prolific.ac/), a crowdsourcing platform designed for academic studies, we assessed all 21 major weight loss diets listed in Wikipedia at the time of this study across six distinct evaluation criteria. Then, using the same source to hire participants, we conducted a user study to compare TDE against Google in discovering personalised weight loss diets. We 
consider Google as a fair yardstick in this case, since people increasingly turn online to find health-related information.

Our results validate that TDE can be used to quickly offer personalised weight loss diets that meet the user's personal needs much in the same way as Google, but faster and without the clutter, advertisements and other identified pitfalls of Google. Finally, we make a technical contribution by providing a considerably improved, re-implemented version of TDE in the plugin repository of the world's most popular online Content Management System, WordPress. Installing the plugin version of TDE is thus a 1-click process, and webmasters can use it for helping their visitors to discover diets and donate data for science.

\section{THE DIET EXPLORER}

\section{Background and Related Work}

Losing weight is challenging. Both a proper and balanced diet, and exercise help, but of those two, diet has been shown to matter more [1]. Yet, as evident from the endless amount of new coming and going dieting fads and advice out there, choosing a diet to begin with is extremely challenging and confusing. To this end, crowdsourcing has emerged as an excellent method to aggregate knowledge that can then be used in recommending suitable and trustworthy options - to basically offer decision support $[4,5]$.

Crowdsourcing has several advantages in decision support, such as collecting large number of potential solutions and evaluating their quality in order to suggest the best ones [6]. Related to this, TaskGenies [7] uses online crowds to create action plans that help people to be more productive in everyday chores. In general, the more specific plans the participants were given, the more productive they became. In a similar vein, PlanSourcing demonstrated how friends and strangers alike may be leveraged to create plans that lead to behavioral changes in form of better personal decisions [8]. This study highlights an interesting characteristic, or benefit, to crowdsourcing: Sometimes it is easier to ask for time-consuming help from strangers than friends. Finally, Hosio et al. discussed a lightweight decision support tool that helps collect solutions to any problem and rate those solutions in terms of different criteria [4].

In this work, we leverage crowdsourcing to assess weight loss diets across a variety of criteria, using a data structure similar to the one described in [4]. In other words, we modelled human-contributed information on diets across a set of criteria by crowdsourcing multiple ratings per each diet-criterion pair. This crowdsourced information repository - a snapshot of the cumulative knowledge of the respondents - was then used to bootstrap a crowdsourced system that allowed users to discover diets that best match their personal preferences, described by a set of optimal/desired criteria values.

However, it is important to note that from a nutrition science perspective, we do not consider if these diets work optimally and for whom. Our interest lies in matchmaking requesters with suitable diets using a crowdsourced approach and investigating how the approach system compares to the contemporary de-facto way of discovering diets: Google.

\section{Implementation}

The Diet Explorer is a lightweight web-based tool that can be embedded on any website using a standard HTML iFrame tag (we describe the Wordpress plugin later). TDE was implemented using HTML, JavaScript, PHP, and MySQL. In essence, it is a crowd- 
powered decision support system [6] that collects data on a question and provides answers by querying the data. As we collect several subjective, independent ratings for each of the available diet-criterion pairs, the resulting knowledge is based on wisdom of the crowd, where the crowd is the people who assessed the pairs. Specifically, in the healthinformation field, a similar approach has been successfully used in the past to recommend and capture data on low back pain treatments [5].

An important consideration with any embeddable tool, such as ours, is the surrounding context, i.e., the website. The context always plays a role in user perceptions. To keep our study design tidy, we deployed TDE as a solo instalment on a blank page online. In other words, there was no context to skew the users' opinions about the tool and its functionality. Instead of a website that would normally introduce the tool, we deployed an additional short splash-screen before loading TDE for the users and set out to examine how TDE succeeds in recommending diets. From the end-users' perspective, TDE consists of two main conceptual stages: one for assessing different weight loss diets and one for discovering personalised diets among all the previously assessed diets. These are separate interfaces, however, and in the study presented in this paper, each participant only used one or the other - not both.

\section{Assessing Diets}

With TDE, every diet is assessed against a set of different criteria, using a slider input element that maps to a numerical scale from 0 to 100 (see Figure 1, screenshot A). This corresponds to how well does the diet in question intuitively perform in terms of the criterion being assessed. The numerical value of the scale is displayed as the user moves the handle and is supplemented with a verbal scale to help the user understand the value. TDE can host an arbitrary number of diets and any arbitrary criteria. A diet in TDE consists of a short title (e.g., "The Paleo Diet"), a longer description of the diet ("The Paleolithic diet is predominantly focused on consuming only foods presumed to have been the only foods available or consumed by humans during the paleolithic era [...]") and a hyperlink to an external information source about the diet (we used Wikipedia links). In a similar vein, a criterion consists of a short title (e.g., "Rapid weight loss potential") and a longer description.

The data model for storing structured subjective knowledge on arbitrary questions has been pioneered earlier in crowdsourcing settings [9]. The individual ratings are independent and from different people, and are therefore useable in estimating the relations

on each diet-criterion pair, based on the theories behind wisdom of the crowd [3]. In other words, the results of the tool are as accurate (or inaccurate) as the people bootstrapping it with their knowledge.

\section{Discovering Weight Loss Diets}

Once data on every diet have been collected, TDE is ready to be used for discovering diets that best match the user's preferences. In the discovery interface, the user indicates personal importance values for the same criteria that were used to assess the diets. All the criteria are again represented with sliders, and the same verbal cues were provided to help users with their thought processes. For instance, in Figure 1 the user seeks to discover diets that, according to the crowd, have very high potential for rapid weight loss and that provide all the nutrients needed for general well-being. The interface also allows the user to reset the 
sliders and start over. The wordings of the criteria were also slightly altered, since assessing diets and indicating the importance of the assessment criterion are two different tasks that require a different choice of words. The original criterion "Monetary cost of the diet" was also inverted here, since when asking users for their appreciation of a characteristic we considered affordability as a more intuitive metric to use.

Clicking the "reveal results" button initiates the query and takes the user to see the bestmatching results. The results interface (depicted in Figure 1-E) lists the five best-matching diets in the left column. The underlying matchmaking algorithm works as follows. Instead of absolute values of the criteria (as was the case in the assessment stage), the user sets importance coefficients to the criteria, ranging from 0 to 100 . The user-set coefficients are then multiplied with the crowdsourced ground truth estimates of each of the diets in the knowledge base. The tally of these scores becomes the diet's final "grade", and the ones with the highest score are the diets that best match the user's requirements. In the interface, the user can also follow a link to the external information source (configurable per each diet) by clicking the icon displayed after each short title. Finally, the interface allows the user to go back and alter the desired configuration to explore how the results change - a process described as "what if analysis" in decision support systems literature [10]. For the study, we also included a button to conclude the task and take the user to a final questionnaire.

For the purposes of this paper, we included an additional step to TDE for collecting personal info (Figure 1, bottom left) to make the user feel like the system considers more attributes than just the criteria values. Specifically, we asked for their weight loss goal, general activity level and biggest problems in losing weight. However, we did not use the personal information in the matchmaking algorithm. This was explained to the user at the end of the study. Furthermore, we included the logos and branding of the authors' institutions (Figure 1, middle, as knowledge of the origin is one of the very initial steps to information trust [11]. 

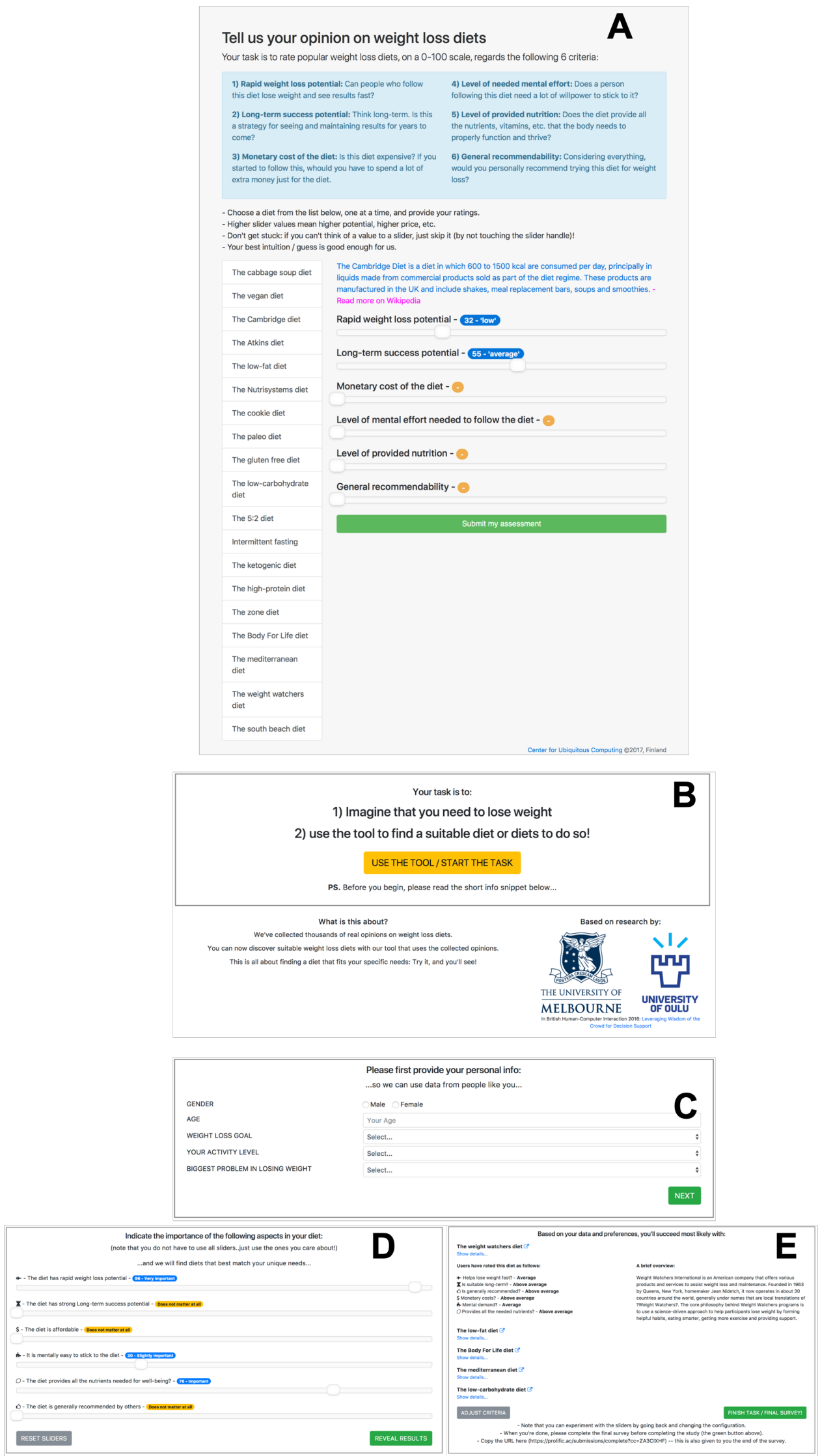
Figure 1. A: the interface to assess diets across different criteria. B: Splash screen used in the experiment. C: Personal information collection screen. D: Personalising a diet by setting ideal criteria configuration. E: The results screen depicting the top-5 matches (diets) and links to read more about them.

\section{THE EXPERIMENT}

We probed TDE's potential with an experiment that required the user to imagine they needed to lose weight and then simply find the best weight-loss diets for themselves by using either TDE or Google. For our between-subjects user study, we recruited two batches of users: one batch used TDE and the other batch used Google. We used Prolific Academic, a crowdsourcing platform specifically designed for academic research, for the recruitment of participants in both conditions. Both conditions ended in a survey online, where the first step was identical to be able to compare TDE to Google in finding diets. The participants using Google were instructed to search online for a minimum of five diets that they identify as the best for their specific needs. The participants were instructed to copy and paste the URLs of their chosen 5 diets to a text area we provided as part of the splash screen, as proof of task completion.

\section{Criteria and Diets Used in TDE}

We acknowledge that the choice of diets and criteria crucially affects the outcome of the experiment. For the study, we simply chose to list all 21 weight loss diets available at Wikipedia at the time of the study, as a fair approximation of the set of most popular diets out there. As for the criteria, the authors examined independently various weight loss forums and magazines online, focusing solely on sites that host user-generated content: Q\&A sites, weight loss and fitness forums, and social (Web 2.0) platforms. The authors then discussed the most commonly encountered criteria that they encountered and concluded to include the following six criteria that were all explained to the participants in more detail:

1. Rapid weight loss potential,

2. Long-term success potential,

3. Monetary cost of the diet,

4. Level of mental effort required to follow the diet,

5. Level of provided nutrition, and

6. General recommendability.

\section{The End User Survey}

The shared first step of the end-user survey, hosted in Google Forms, that participants in both conditions were required to fill in started with a 7-point Likert Scale [12] with the following items:

- General trustworthiness of the obtained results,

- Relevance of the results to the used search parameters,

- Considering everything, how satisfactory were the results, for the task at hand,

- How rapid was the process, and 
- The likelihood of the user choosing one of the discovered options as the first choice of diet, if the user was supposed to start dieting for weight loss, right now.

The items were labelled from "not at all" to "extremely", and in addition to the Likert Scale we asked for the participant's demographic data. The second step of the survey was condition-specific. For the participants who used TDE, we asked open-ended questions on which criteria (if any) are missing from TDE, how exhaustive/sufficient the tool covers the list of diets, if the users would recommend TDE to a friend who wants to lose weight, any positive or negative aspects observed in TDE, and finally, suggestions to improve the tool.

As mentioned earlier, for the participants who used Google the first part of the survey was shared, and the second part of the survey focused open-endedly on uncovering the specific pros and cons of Google in finding weight loss diets.

\section{RESULTS}

Before we could study finding diets with TDE, we needed to bootstrap TDE, i.e., populate the knowledge base on the diets chosen by us. For this purpose, we recruited 70 participants (47 female, 22 male, 1 other, average age 31.3) from Prolific Academic. The participants provided 8607 ratings for the diets (average of 68.3 ratings per each pair). In similar crowdsourced systems (e.g., [5]), a far smaller participant number was shown to provide reliable results, so we argue 70 is an adequate number for us in this case. While a more exhaustive analysis of the assessment is out of scope of this paper, in Table 1 we present the three top- and bottom-rated diets (based on absolute numerical values acquired in the assessment stage) per criteria.

Table 1: A summary of the best and worst-rated diets in the experiment

\begin{tabular}{lcccccc}
\hline & Rapid & Long-term & Cost & $\begin{array}{c}\text { Mental } \\
\text { Effort }\end{array}$ & $\begin{array}{c}\text { Nutrition } \\
\text { Levels }\end{array}$ & Recommendability \\
\hline Top-3 & $14(82.2)$ & $15(72.2)$ & $6(74.3)$ & $21(92.4)$ & $6(66.2)$ & $21(68.6)$ \\
& $12(78.5)$ & $21(64.7)$ & $23(72.9)$ & $17(75.8)$ & $21(63.5)$ & $6(64.1)$ \\
& $9(75.0)$ & $10(63.1)$ & $7(71.6)$ & $11(75.0)$ & $4(63.5)$ & $15(60.3)$ \\
Bottom-3 & $17(39.8)$ & $24(24.4)$ & $12(22.6)$ & $21(51.3)$ & $12(25.5)$ & $5(25.4)$ \\
& $21(32.4)$ & $14(15.8)$ & $16(20.7)$ & $17(50)$ & $11(12.4)$ & $14(7.4)$ \\
& $11(2.6)$ & $11(1.6)$ & $14(5.3)$ & $11(10)$ & $14(5.8)$ & $11(2.4)$ \\
& & \multicolumn{5}{c}{ LEGEND } \\
\hline
\end{tabular}




\begin{tabular}{llll}
\hline 4 & The Nutrisystems diet & 15 & The weight watchers diet \\
5 & The cookie diet & 16 & Intermittent fasting \\
6 & The Body For Life diet & 17 & The gluten free diet \\
7 & The paleo diet & 18 & The ketogenic diet \\
8 & The vegan diet & 19 & The high-protein diet \\
9 & The Atkins diet & 20 & The low-fat diet \\
10 & The low-carbohydrate diet & 21 & The mediterranean diet \\
11 & The junk-food diet & 22 & The zone diet \\
12 & The cabbage soup diet & 23 & The Cambridge diet \\
13 & The south beach diet & 24 & The 5:2 diet \\
14 & The breatharians diet & & \\
\hline
\end{tabular}

\section{TDE vs. Google in Discovering Diets}

We recruited 41 participants (22 female, 19 male, average age 32.9) from Prolific Academic to discover diets with our tool. 37 of the participants indicated that they actually want to lose weight, making them a suitable audience for the evaluation since such people are among the actual intended audience that we hope to be able to eventually serve with the tool. Using manually paid bonuses, we ensured that everyone was paid 12-15 GBP per hour for the work, to adhere to fair pay policies of Prolific Academic.

To compare the process with finding diets using Google, we recruited 40 participants (23 female, 17 male, average age 30.3) from Prolific Academic to find diets using Google, ensuring that these were new participants. Of these 40,31 indicated that they want to lose weight. For the participants finding diets with Google, we used the same payment scheme as with TDE.

In analysing the result data, we found no evidence of significant differences between TDE and Google in terms of:

- Trustworthiness of the discovered diets: averages 5.4 and 5.3, respectively

- Their relevance to the used search parameters, i.e. criteria in TDE and search terms in Google: 5.3 and 5.6

- Overall satisfaction with the discovered diets: 5.4 and 5.6

- Acceptability of the results, i.e., if the users would likely start with one of the discovered diets if they were to start losing weight now: 5.8 and 6.0

In terms of perceived speed of the task, however, there was a significant difference in perception, as TDE was perceived as faster than Google in discovering diets with (6.2 and 5.1; Wilcoxon Rank-Sum Test, $\mathrm{p}<.01$ ). To summarise, TDE comes close to Google in performance across all other measured items and is better in the perceived speed of the search task. Indeed, only 5 of the 41 participants would not recommend TDE for their friends who want to lose weight (whereas 28 answered yes, and 8 answered maybe).

\section{Qualitative Insights}

Two of the paper's authors first reduced the result to three clear overarching themes, namely pros and cons of the two approaches and improvement suggestions to TDE. A third author was invited to verify the classification, after which we moved all the items to a shared spreadsheet online and iterated the labeling process until all relevant comments belonged to one or more subthemes.

First, we noticed that participants frequently mentioned the clutter and advertisements 
present when using Google. While we were not surprised to see comments about the excessive amount of advertisements encountered with Google (and online in general - our participants only started with Google and ended up on 3rd party sites), the interesting finding here is how useful the ads seemed to be for certain participants:

"I find the promoted ads at the top of searches are actually quite useful in searching for new diet plans and they often show the best value options"

The ads were seen as fairly accurate and actually contribute to the search rather than always seen as negative. This opinion was split, however, and more people still perceived ads as more harmful than good:

"lots of adverts and misleading diet offers"

"There's SO MANY!"

Overall, participants were aware of all the false promises and companies "trying to get your money". Several participants were also concerned about finding potentially unhealthy or outright harmful solutions, such as diet pills and other "snake oils" that are, in reality, some of the most lucrative dieting products out there from the vendor's standpoint. During the past years, Google itself has done a laudable job in banning such products and their advertisement campaigns. The sites where users end up via Google are, however, a different playground. There, users are still exposed to all types of non-legit dieting and weight loss offers. In other words, Google is practically helpless in this matter.

Overall, however, Google's search capabilities were found as impressive:

"It's a good search engine capable of helping narrow down a large number of results if you're really specific in what you're looking for"

The one thing that was clearly appreciated by TDE users the straightforward approach of discovering diets to consider. Responses such as "quick" and "easy to use" occurred frequently:

"I like how simple and easy it is. Also, I like the fact that you get multiple options"

Two participants even reported finding diets they had not heard of before, and one commented that she is even going to try it after participating in the study. As for key problems with TDE, most often participants would have liked to see more information on the diets (e.g., pork-free or vegetarian recipes that would go together with the diet):

"There was no accommodation for allergies or personal/religious restrictions. I don't want to be recommended a high-meat diet if I'm vegetarian, for example”

Other participants were disappointed by not finding diets that they enjoy eating (such as curry) and took a very hedonistic stance in dieting ("it looks as if there would not be any enjoyment left in eating"). Such insights are great in understanding what goes on in the minds of people looking for diet options and thus in developing the next versions of TDE. 


\section{TDE as a Re-Implemented Tool for Science}

While controlled experiments have their uses, we argue that the online equivalent of an uncontrolled "field study", i.e., placing the tool as part of the existing fabric of the Web, is a currently under-exploited opportunity in health-information related experiments. Such an approach, if executed with care, will produce results with high ecological validity and help reach people who otherwise would be out of reach of academic studies. With this in mind and informed by the qualitative findings from our experiment, we converted TDE to a WordPress plugin and added three new features to it: a featured diet option, a promotion box, and a data capture feature (detailed below). WordPress is the world's most popular Content Management System and currently powers tens of millions of websites online according to estimates over $30 \%$ of all blogs and websites in existence. The new features are only available in a commercial version of the plugin, but we offer any non-profit academic organization a free-forever license for the premium version. The new features are as follows.

Featured Diet Option; Similar to native advertising online, we provide a placeholder where the manager using the plugin can control what is shown as the fifth discovered diet option. If no featured diet is provided in the plugin settings, in the WordPress admin area, or if this option is turned off the plugin simply shows the fifth best-matching option (Figure 2-E).

Promotion Box; Second, we provide a promotional area surrounded by a clear visual border in the results screen. The promotion box can contain both text and a link. As depicted in Figure 2-E, the text is placed before a big yellow button that opens the URL in a new browser tab. Here, any additional resources relevant to the user's interests can be promoted. In the case of scientific studies, this area could be used to, for example, deploy different conditions in a study, direct the user to a survey or indeed simply invite the user to another study by using the plugin as the magnet to get the user interested in exploring different options in a fun way.

User Data Capture and Follow-Up; Finally, the most powerful feature of the plugin, depicted in Figure 2-F and accessible through a button in the results screen (Figure 2-E) is the user data capture feature. This screen simply asks for the user's name (or nickname) and an email address. The plugin settings screen (Figure 2-A) allows for setting a webhook destination, where all the data is sent when the users request for "more help". Naturally, for academic purposes these texts can be changed to reflect the study's purpose. Once the user requests for more help, or follow-up, the plugin sends to the webhook in a JSON payload:

- The responses that the user gave in the personal data collection stage (Figure 2-C)

- The used search parameters (criteria configuration)

- The user's name and email address

- The top results that the used criteria yielded

This feature can be used in automated fashion to respond to user's requests for more details on the chosen diet - a key finding in our qualitative results. Further, this feature is particularly useful in many types of experiments, including follow-up studies where the user data is inserted into a CRM and the user is later invited back to e.g. fill in periodical 
surveys or to participate in new studies.

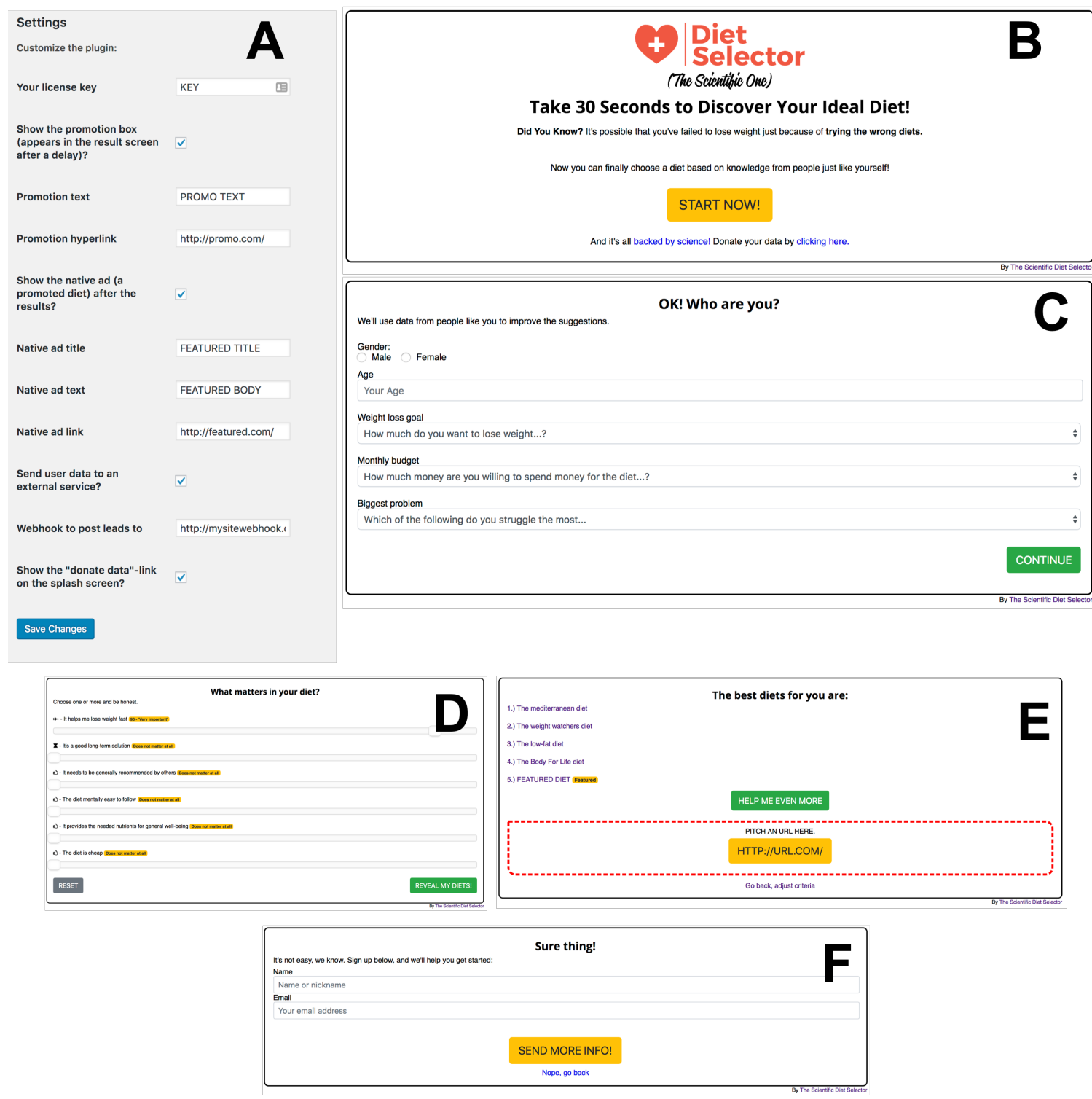

Figure 2. The Diet Explorer (rebranded as The Scientific Diet Selector), re-implemented as a WordPress plugin. A: the back-end settings panel to customize content containers and set the webhook (or switch the customizable features on/off). B: in intro screen, with links to read more and donate data to science. C: User data collection form. D: The criteria selection, i.e. determining the characters of ideal diets. E: The results screen, with a featured customizable diet placeholder and a promotion box. F: Lead collecting form, for integration with any backend or CRM for follow-up studies and surveys.

\section{DISCUSSION AND CONCLUSION}

In the bigger picture of obesity, tools such as ours are not a panacea. However, given the magnitude of the problem, no stone should be left unturned in exploring how to offer 
suitable lifestyle choices to people. And discovering diets is only one part of the puzzle. Diets differ, there is no clear academic consensus of what diets should one follow, and a diet that works for one might not work for the other. Nevertheless, TDE was created to let people discover and consider diets that may fit their needs, based on assessment by real people. By design and choice, we detach from the scientific debate of the diets' effectiveness, but provide users a chance to discover a set of diets that a crowd (be it whatever it is) has assessed as suitable in terms of the search parameters used (the criteria).

We consider our results not as perfect but promising. Using only the diets from a ranked list on Wikipedia and a public source for participants to assess them, we were able to bootstrap TDE to a state where the discovered diets compared relatively well against the ones people discovered with Google. Moreover, TDE was seen as a rapid and straightforward tool for this. And for the very same reason, we see TDE as a potential platform for conducting academic research and highlight the plugin as a prominent output of this work.

\section{Design Opportunities}

The lack of details and specific information in the diets was perhaps the one thing that bothered the users of TDE the most. As the concept relies on crowdsourcing, we are exploring how to use the plugin to support users themselves contributing extra information such as food plans, links to online resources, or gluten-free or vegan options of the same diet. Most likely this will require exploring incentive mechanisms as well and carefully considering where this data collection happens (either on a 3rd party website or within the plugin itself).

Second, while in this study with TDE we deliberately detached from scientific debate on the diets and their efficiency in losing weight, we could bootstrap several versions of the tool. Several end-users mentioned that the diets were all known to them already, and more could be added. The study here had a limitation in that it only considered diets from Wikipedia. Currently, we manage the diet knowledge base in a centralized fashion, but we already allow adding new diets to the database. However, we lack means to derive a solid academic understanding of the diets. Therefore, hiring a pool of actual nutrition/dieting experts to rate the diets would offer great value to complement the peer-sourced knowledge. Further, we need to work on ensuring the tools' scalability: how many users can we serve, should the tool gain wide adoption?

Finally, an addition we already support but do not offer as a service in the plugin is a pre-created follow-up sequence that motivates the users to follow a diet - to stick to the chosen diet, as choosing a weight loss diet really is just the start. Stickiness was also suggested to be added as a criterion by several participants. To this end, researchers can implement a motivational follow-up sequence that the user can subscribe to, directly from the plugin by using the built-in webhook integration feature.

\section{Conclusion}

We presented The Diet Explorer and our initial study with it. We find TDE as a promising system for discovering diets that provides a fair alternative for Google especially for people new to dieting and who just want to quickly get an overview of diets that may be suitable. As the most important contribution of this paper, we highlight the WordPress plugin available in the public repository for the community to use. 


\section{REFERENCES}

1. W.C.M. Hamilton, et al., "A meta-analysis of the past 25 years of weight loss research using diet, exercise or diet plus exercise intervention," International Journal of Obesity, vol. 21, no. 10, 1997, pp. 941; DOI doi:10.1038/sj.ijo.0800499.

2. Y.M. Chan and H. Huang, "Weight Management Information Overload Challenges in 2007 HINTS: Socioeconomic, Health Status and Behaviors Correlates," Journal of Consumer Health on the Internet, vol. 17, no. 2, 2013, pp. 151-167; DOI 10.1080/15398285.2013.780540.

3. F. Galton, "Vox populi (the wisdom of crowds)," Nature, vol. 75, 1907, pp. 450451.

4. S. Hosio, et al., "Leveraging Wisdom of the Crowd for Decision Support," Proc. Human Computer Interaction Conference, 2016, pp. 1-12.

5. $\quad$ S.J. Hosio, et al., "Crowdsourcing Treatments for Low Back Pain," Proc. Proceedings of the $2018 \mathrm{CHI}$ Conference on Human Factors in Computing Systems, ACM, 2018, pp. 276.

6. C.-M. Chiu, et al., "What can crowdsourcing do for decision support?," Decision Support Systems, vol. 65, 2014, pp. 40-49; DOI 10.1016/j.dss.2014.05.010.

7. N. Kokkalis, et al., "TaskGenies: Automatically Providing Action Plans Helps People Complete Tasks," ACM Trans. Comput.-Hum. Interact., vol. 20, no. 5, 2013, pp. 27 21-27 25; DOI 10.1145/2513560.

8. E. Agapie, et al., "PlanSourcing: Generating Behavior Change Plans with Friends and Crowds," Proc. Conference on Computer-Supported Cooperative Work \& Social Computing, ACM, 2016, pp. 119-133.

9. J. Goncalves, et al., "Eliciting Structured Knowledge from Situated Crowd Markets," ACM Transactions on Internet Technology, vol. 17, no. 2, 2017; DOI 10.1145/3007900.

10. S. Alter, "Decision support systems: Current practice and continuing challenges. Reading, Massachusetts: Addison-Wesley Publishing Co., 1980, 316 pp.," Behavioral Science, vol. 27, no. 1, 1982, pp. 91-92; DOI 10.1002/bs.3830270109.

11. J.R. Nurse, et al., "Information quality and trustworthiness: a topical state- ofthe- art review," Proc. The International Conference on Computer Applications and Network Security (ICCANS) 2011, IEEE, 2011, pp. 492-500.

12. R. Likert, "A technique for the measurement of attitudes," Archives of psychology, 1932. 